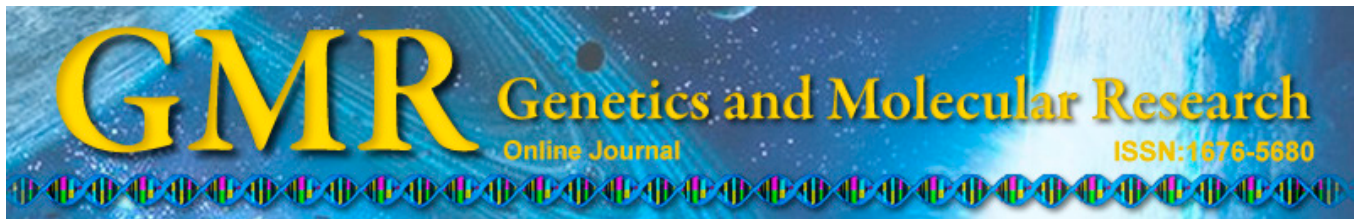

\title{
Clinical and pathological features and treatment of AIDS-related cutaneous Kaposi's sarcoma in Chinese Han patients
}

\author{
X.-K. Zheng ${ }^{1}$, S.-H. Lu ${ }^{2}$, J.-F. Liu ${ }^{1}$ and Y.-R. Lai ${ }^{1}$ \\ ${ }^{1}$ Department of Pathology, The First Affiliated Hospital, \\ Sun Yat-Sen University, Guangzhou, China \\ ${ }^{2}$ Department of Dermatology-Venereology \& AIDS, \\ Guangzhou Eighth People's Hospital, Guangzhou, China \\ Corresponding author: Y.-R. Lai \\ E-mail: laiyingrong_lyr@163.com
}

Genet. Mol. Res. 14 (2): 6830-6837 (2015)

Received September 17, 2014

Accepted January 14, 2015

Published June 18, 2015

DOI http://dx.doi.org/10.4238/2015.June.18.25

ABSTRACT. This retrospective study aimed to observe the clinicopathological features and immunological phenotypes, and explore effective treatment and prognosis for 12 Chinese Han patients with acquired immunodeficiency syndrome-related cutaneous Kaposi's sarcoma. All 12 patients were human immunodeficiency virus-positive, and underwent the standard highly active antiretroviral therapy (HAART). Skin lesions mainly presented as purple, or rufous papules, or plaques; skin biopsy showed diffuse or flaky infiltration of spindle cells, active proliferation of slit-like vasculature, erythrocyte exudation, hemosiderin deposition, and inflammatory cell infiltration. Immunohistochemical analysis showed the expression of Ubiquitin C-terminal hydrolase L1 $(+)$, and CD31 $(+)$ in T-cells; factor VIII $(+)$ and HHF-35 $(+)$ in the proliferating vascular endothelial cells; vimentin $(+)$ and $\mathrm{S}-100$ protein $(-)$ in the vessel wall; and CD34 (+++) in the spindle cells of 6 cases, with 1 case of negative CD34 expression. Four patients with confined lesions underwent surgery and microwave therapy, and received a favorable prognosis. Two patients with limited lesions underwent microwave 
therapy, and the lesions subsided. Of six patients with widely distributed sarcomas, five underwent microwave therapy and one received combined chemotherapy; five attained significant efficacy, and one died. There were no significant differences in the clinicopathological features and immunological phenotypes between the Chinese Han patients and those from other populations. Along with basal HAART, patients in early stages, with sarcomas $<2 \mathrm{~cm}$ in diameter should undergo surgery and microwave therapy, while patients with sarcomas $>2 \mathrm{~cm}$ in diameter should undergo chemotherapy and microwave therapy.

Key words: Acquired immunodeficiency syndrome; Pathological features; Highly active antiretroviral therapy; Kaposi's sarcoma; Therapeutic effect

\section{INTRODUCTION}

Kaposi's sarcoma (KS) is a type of multiple hemorrhagic sarcomas caused by an infection with the Kaposi's sarcoma-associated herpes virus/human herpes virus- 8 ; it is common in the African and Western European populations, especially in patients infected with the human immunodeficiency virus (HIV). Although it is relatively less prevalent in the Chinese Han people, there is clinical value in exploring its pathology, diagnosis, and treatment.

\section{MATERIAL AND METHODS}

\section{Materials}

\section{Clinical data}

The 12 Chinese Han patients with acquired immune deficiency syndrome (AIDS)-related cutaneous KS were in-hospital patients at Guangzhou Eighth People's Hospital, admitted from October 2007 to October 2010. All patients were anti-HIV positive by screening and confirmatory test (by Disease Prevention and Control Center of Guangdong Province or Guangzhou City, AIDS confirmatory testing laboratory) and diagnosed with AIDS-related KS by skin biopsy.

\section{Reagents}

The three-color kit for the detection of cluster of differentiation (CD)3+, CD4+, and CD8+ T lymphocytes was provided by Becton, Dickinson and Company, USA. The primary antibodies for immunohistochemistry [anti-factor VIII antibody, anti-muscle actin antibody (HHF-35), anti-vimentin antibody, anti-CD31 antibody, anti-CD34 antibody, S-100, and antiUbiquitin carboxyl-terminal hydrolase L1 (UCHL-1) antibody] were supplied by The Dallas Area Kitefliers Organization (DAKO). The EnVision secondary antibody detection system for immunohistochemistry was purchased from Gene Company. Hematoxylin and eosin (H\&E) were purchased from SIGMA Company, USA.

\section{Methods}

The number of CD3+, CD4+, and CD8+ T lymphocytes in blood samples was detected 
by flow cytometry (BD FACS Calibur, USA). Biopsy specimens were fixed in $4 \%$ neutral formalin, embedded in paraffin, and sectioned into slices of $4 \mu \mathrm{m}$ thickness. Sections were stained with HE, and the EnVision two-step staining method was used for immunohistochemistry.

\section{Therapeutic regimens}

All 12 Chinese Han patients with AIDS-related KS underwent treatment with highly active anti-retroviral therapy (HAART), which included the therapeutic regimen of lamivudine $(3 \mathrm{TC})+$ stavudine (d4T) + Kaletra, or Kaletra or efavirenz (EFV) or nevirapine (NVP). For patients in the early stage with limited skin lesions, the tumor was surgically removed when it was smaller than $2 \mathrm{~cm}$ in diameter, and patients underwent basal HAART, with microwave therapy as auxiliary treatment; when tumor size was greater than $2 \mathrm{~cm}$ in diameter, patients received supplementary microwave therapy with basal HAART. Conversely, for patients with widely distributed skin lesions, microwave therapy and/or chemotherapy was applied with basal HAART. Of all 12 patients, five received auxiliary microwave therapy only, one received supplementary surgery alone, while five patients underwent surgery with microwave therapy, and only one patient received supplementary microwave therapy and chemotherapy simultaneously.

\section{Prognostic analysis}

A follow-up of 1-4 years was carried out to evaluate the prognosis of these 12 patients. Outcomes of the patients (improved, relapse, death) were defined as follows: improved: significant improvement in clinical symptoms, most or all of the lesions subsided; relapse: the appearance of new skin lesions in patients at the same site or in new sites; or death: patient died during the time span.

\section{RESULTS}

\section{Clinical manifestations of patients}

All 12 patients were HIV positive and received a pathological diagnosis of KS. Of the 12 patients, seven had symptoms of fever and pulmonary infection, six patients showed enlargement of the submandibular or posterior auricular lymph nodes, and two patients showed inguinal lymph node enlargement. Six patients had limited skin lesions, located respectively on the part of the left hand between the thumb and the index finger (Figure 1A), the right labium minus and vulva back, the lower one-third part of left anterior tibia (Figure 1B), and on the legs and buttocks. These skin lesions were dark red, round uplifted nodules or bumps. The skin lesions were of the following sizes: the lesions on the left hand and the right labium minus were $0.8 \times 0.8 \mathrm{~cm}$, the lesion on the vulva back was $0.5 \mathrm{~cm}$ in diameter, the lesion on the buttocks was $1.5 \mathrm{~cm}$ in diameter, and the lesion on the anterior tibia was about $2.5 \mathrm{~cm}$ in diameter. All these lesions had a rough surface, clear edge, minor desquamation, and no touch tenderness. Conversely, six patients had widely distributed skin lesions on the hand, foot (Figure 1C), limbs, neck, jaw and body trunk, which manifested as solid dark red or purple papules and plaques or nodules, which differed in sizes and shapes. The amount of CD3+, CD4+, and $\mathrm{CD} 8+\mathrm{T}$ lymphocytes in these skin lesions and their expression of CD4+/CD8+, and treatment options and prognosis are shown in Table 1. 

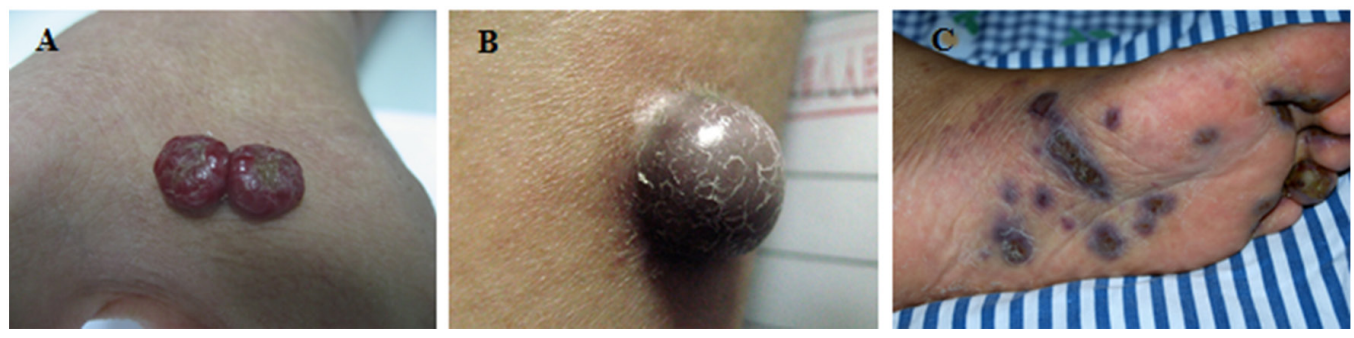

Figure 1. Skin lesions in the patients with cutaneous Kaposi's sarcoma. A. Purple and round papules on the dorsum of the hand; B. purple and round nodules on the left shank; C. dark plaque and nodules on the sole of the foot.

\section{Biopsy and immunohistochemistry results}

Skin lesions were collected from the 12 patients by skin biopsy and dehydrated, embedded, and sectioned for regular HE staining, special histochemical staining, and immunohistochemistry. After HE staining, all slices showed flaky or diffusely distributed spindle cells, highly proliferative cleft-like microvasculature, with exudation of erythrocytes into interstitial spaces (Figure 2A) and chronic inflammatory cell infiltration within the dermis; special histochemical staining showed hemosiderin $(+)$, fungal staining $(-)$, and acid-fast staining (-). Moreover, immunohistochemistry of the 12 cases was performed (confirmation was provided by two independent professors) to detect protein expression of Factor VIII, HHF-35, vimentin, CD31, CD34, S-100, and UCHL-1; the results showed expression of UCHL-1(+) in T lymphocytes, CD31(+) in the adjacent area, Factor VIII $(+)$ in the proliferative vascular endothelial cells (Figure 2B), and HHF-35 (+), vimentin $(+++)$ (Figure $2 \mathrm{C}), \mathrm{S}-100$ protein (-) in the vessel wall. In six cases, CD34 was highly expressed (+++) (Figure 2D) in spindle cells; however, in one case there was no expression of CD34 (-) in spindle cells. Details of the immunohistochemistry results, diagnosis, and clinical staging are provided in Table 2 .

\section{Prognosis of patients}

All 12 patients were diagnosed with AIDS-related cutaneous KS and underwent HAART, with surgical removal, microwave therapy, or chemotherapy as auxiliary treatments. Patients were followed up for 1-4 years. The patient with the skin lesion in the left thumb area (tumor size $0.8 \mathrm{~cm}$ in diameter) relapsed one week after surgical removal and continued antiviral therapy for 3 months until the lesions subsided. During the 4-year-follow-up afterwards, no recurrence was observed. The patient with the lesion on the labium minus (tumor size $0.8 \times 0.8$ $\mathrm{cm}$ ) underwent surgery and microwave therapy, and the prognosis was favorable. The patient with skin lesions on the left anterior tibia and legs (tumor size $2.5 \mathrm{~cm}$ in diameter) showed lesion subsidence after microwave therapy. Six patients with widely distributed skin lesions all received microwave therapy with basal HAART, with or without surgery and chemotherapy. During the one-year follow-up, most skin lesions had subsided in five cases; however, one patient died because of end-stage AIDS cachexia and circulatory failure. Table 1 shows the details of treatment and prognosis for all patients. 


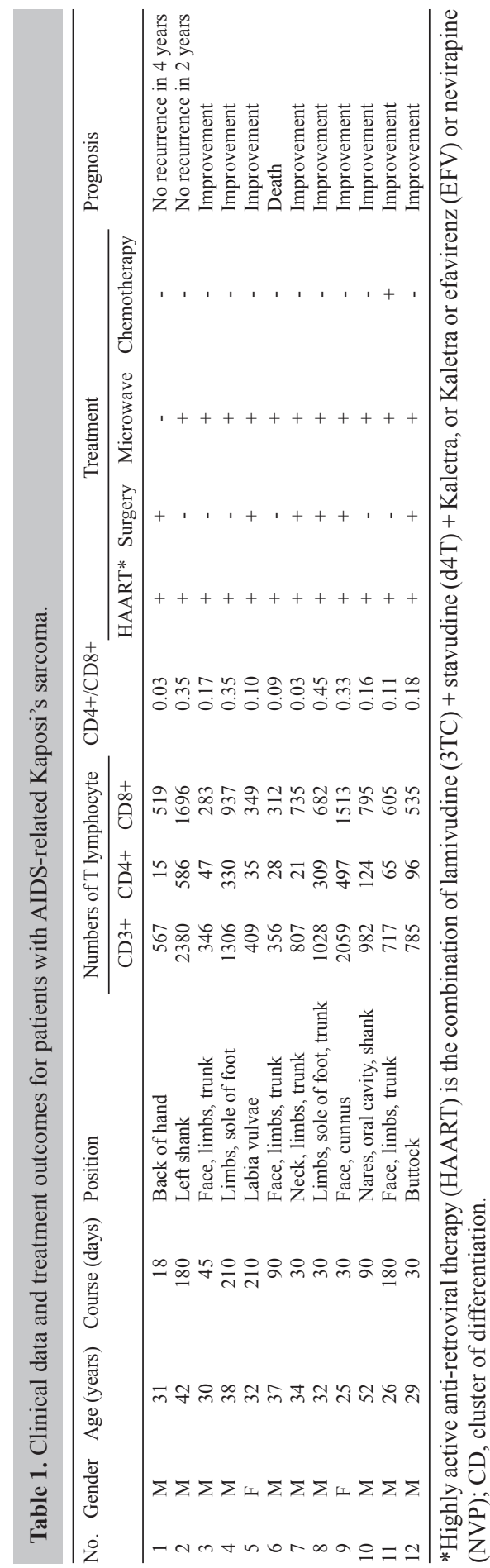



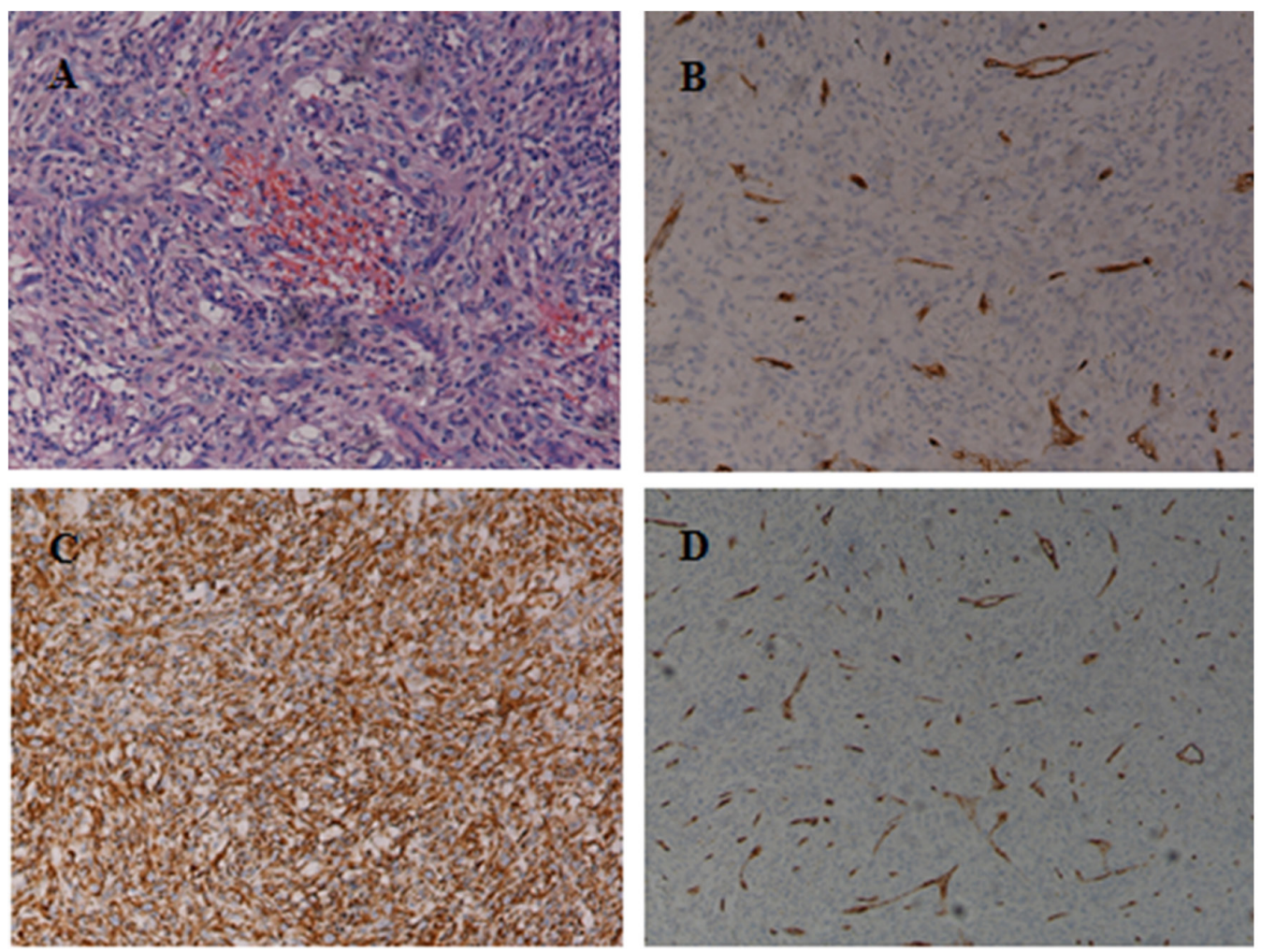

Figure 2. Histological and immunohistochemical analysis of cutaneous Kaposi's sarcomas. A. Active proliferation of slit-like blood vessels and the effusion of red blood cells in Kaposi's sarcoma (HE staining, 100X); B. expression of Factor VIII by immunohistochemical staining of the sarcoma section; C. expression of vimentin by immunohistochemical staining; D. expression of CD34 by immunohistochemical staining (100X).

Table 2. Immunohistochemical analysis and clinical stage of the patients with AIDS-related Kaposi's sarcoma.

\begin{tabular}{|c|c|c|c|c|c|c|c|c|c|}
\hline \multirow[t]{2}{*}{ No. } & \multicolumn{7}{|c|}{ Results of immunohistochemistry } & \multirow[t]{2}{*}{ Clinical stage } & \multirow[t]{2}{*}{ Prognosis } \\
\hline & Factor VIII & HHF-35 & Vimentin & CD31 & CD34 & S-100 & UCHL-1 & & \\
\hline 1 & ++ & ++ & ++ & + & +++ & - & + & II & No recurrence in 4 years \\
\hline 2 & + & ++ & ++ & + & ++ & - & + & III & No recurrence in 2 years \\
\hline 3 & ++ & + & ++ & + & +++ & - & + & IV & Improvement \\
\hline 4 & ++ & + & + & + & ++ & - & + & IV & Improvement \\
\hline 5 & ++ & + & ++ & + & +++ & - & + & IV & Improvement \\
\hline 6 & ++ & ++ & +++ & + & ++ & - & + & $\mathrm{V}$ & Death \\
\hline 7 & ++ & + & +++ & - & +++ & - & + & IV & Improvement \\
\hline 8 & + & + & ++ & + & ++ & - & + & III & Improvement \\
\hline 9 & ++ & ++ & ++ & + & +++ & - & + & III & Improvement \\
\hline 10 & ++ & + & + & - & +++ & - & + & III & Improvement \\
\hline 11 & + & + & ++ & + & ++ & - & + & IV & Improvement \\
\hline 12 & + & + & + & + & - & - & + & IV & Improvement \\
\hline
\end{tabular}

UCHL-1 = Ubiquitin carboxyl-terminal hydrolase L1; CD = cluster of differentiation. 


\section{DISCUSSION}

Kaposi's sarcoma occurs mainly in African and Western countries, and presents in four different epidemiological forms: classic, endemic, iatrogenic, and AIDS-associated. The first three forms of KS often lack a history of HIV infection, while patients with AIDS-related $\mathrm{KS}$ are HIV positive. In China KS is mainly observed in the Uighur population from the Xinjiang region, with obvious ethnic specificity. Most KSs appear on the limbs, in the form of skin lesions. Initially, they are blue-red nodules or spots $2-5 \mathrm{~cm}$ in diameter, often accompanied by swelling of the affected limb, polypoid-uplift, and granulomatous tissue-type capillary hemangioma, generally with long duration. In KS, there are numerous regular clefts, lined with slender, mild atypical cells; red blood cells, hemosiderin phagocytes, and varying numbers of spindle cells (some with large and irregular nucleus or nuclear atypia) may be observed within the clefts. Our study has confirmed all the pathological features described above. Moreover, no significant differences in the aforementioned pathological features were observed between the Uygur and Kazak patients from China's Xinjiang region (Xu et al., 2005) and those from African countries. In the present study, immunochemistry analysis of the patient's skin lesions showed positive expression for CD31, CD34, and vascular Factor VIII, indicating the vascular endothelial cellular origin; and negative expression for S-100 protein (-), UCHL-1 (+) in T cells indicated that the proliferating cells did not originate from melanocytes or lymphocytes, excluding malignant melanoma, lymphoma, and other diseases. The fungus was not cultured from the scurf, the Mycobacterium tuberculosis-related examination was negative, and the tumor marker examination showed no abnormalities. Based on the clinical manifestations, lesion-tissue biopsy results, immunohistochemistry results, and other laboratory tests, the patients were diagnosed with AIDS-related cutaneous KS.

Kaposi's sarcoma is one of several complications in AIDS patients who suffer from severe immune deficiencies. An HIV infection can alter the natural history of KS, increasing its chances of occurrence in young people, leading to its rapid progression, high mortality, and difficulty in treatment. Cattelan et al. (2005) reported that HAART may improve the natural history of AIDS-related KS by inhibiting HIV replication effectively, minimizing the viral load in the blood of the patients, and improving their immune function. After HAART, most patients with AIDS-related KS undergo obvious lesion subsidence. Some patients may experience complete eradication of skin lesions and long-term remission. Moreover, this improvement in symptoms was associated with an increase in the CD4 + T lymphocytes, with a concurrent decline in the replication of RNA in HIV-I (Lebbe et al., 1998; Cattelan et al., 2005). Few domestic studies have focused on the treatment of AIDS-related Kaposi's sarcoma, and the number of cases is very limited. Shi et al. (2013) used HAART combined with taxol in the treatment of one Han patient with AIDS-related KS and reported a good prognosis. Zhang et al. (2009) reported that of 5 Xinjiang Uygur cases with AIDS-related Kaposi's sarcoma treated with a combination of HAART with biological therapy, only one patient survived. Tang et al. (2012) observed that patients with AIDS-related KS treated with immunotherapy and/or chemotherapy with basal HAART received a better prognosis than 3 patients who received HAART alone. However, the patient's race was not apparent in this study.

In the present study, we analyzed the clinical and pathological features in 12 Chinese Han patients with AIDS-related Kaposi's sarcoma, and implemented different therapeutic regimens based on the location, size, and extension of skin lesions in each patient. Along with a basal HAART regimen, four patients with smaller tumors (tumors smaller than $2 \mathrm{~cm}$ in 
diameter) received microwave therapy after surgical removal of the tumor, with a favorable prognosis. Two patients with larger skin lesions on the anterior tibia or legs (tumors of $\sim 2.5$ $\mathrm{cm}$ in diameter) received microwave therapy and experienced lesion subsidence. Of the six patients with widely distributed skin lesions who received supplementary microwave therapy and chemotherapy, five experienced skin lesion remission, and one died. Based on these data, we propose that the prognosis of AIDS-related KS is associated with the size, location, and range of skin lesions. Moreover, our study found ( $\mathrm{Lu}$ et al., 2011) that of all the 12 patients, five patients showed an increase in the number of CD4+ T lymphocytes and a decrease in the copy number of HIV-I RNA after treatment. Conversely, one patient who ultimately died had shown decreased numbers of CD4+ T lymphocytes; therefore, we conclude that in this group of patients, the prognosis of AIDS-related KS correlated with the level of CD4+ T lymphocytes and the decrease in HIV-I RNA replication after therapy.

To summarize, we propose that for patients with AIDS-related KS who have a limited number of skin lesions, small lesions should be surgically removed, and for both small and larger lesions microwave therapy should be applied along with basal HAART. Furthermore, for patients with widely distributed skin lesions, microwave therapy and surgery and/ or chemotherapy is recommended in addition to the basic HAART regimen, and should be considered based on the individual condition of each patient. While HAART is indisputably an essential treatment for AIDS-related KS, the selection of combined therapy should be based on the location, size, and range of skin lesions: patients in the early stages, with sarcomas $<2$ $\mathrm{cm}$ in diameter should be treated with surgery and microwave therapy, while patients with sarcomas $>2 \mathrm{~cm}$ in diameter should receive chemotherapy and microwave therapy.

\section{ACKNOWLEDGMENTS}

Research supported by the Guangdong Province Science and Technology Plan Projects (\#2006B360300002).

\section{REFERENCES}

Cattelan A, Calabrò M, De Rossi A, Aversa SM, et al. (2005). Long-term clinical outcome of AIDS-related Kaposi's sarcoma during highly active antiretroviral therapy. Int. J. Oncol. 27: 779-785.

Lebbe C, Blum L, Pellet C, Blanchard G, et al. (1998). Clinical and biological impact of antiretroviral therapy with protease inhibitors on HIV-related Kaposi sarcoma. AIDS 12: F45-F49.

Lu SH, Tang XP, Lai YR, Hu RX, et al. (2011). Clinical analysis of AIDS-related Kaposi's sarcoma in Chinese Han people. Chin. J. Infect. Dis. 29: 375-378.

Shi JC, Yu JH, Qu B, Zhang BH, et al. (2013). AIDS-related Kaposi's sarcoma treated with taxol in 1 case. Chin. J. AIDS STD 19: 605 .

Tang GP, Wu AP, Pa LD, Ding Y, et al. (2012). Report of seven cases with AIDS complicating Kaposi's sarcoma. Chin. J. AIDS STD 18: 630.

Xu YM, Pu XG and Li F (2005). Clinical and pathological profiles of 43 cases of Kaposi's sarcoma in Xinjiang Uygur autonomous region. China J. Leprosy Skin Dis. 21: 685-687.

Zhang L, Pei YL, Tursun E, and Maenongr E (2009). Clinical analysis of 25 patients with Kaposi's sarcoma. J. Mod. Oncol. 17: 944-946. 